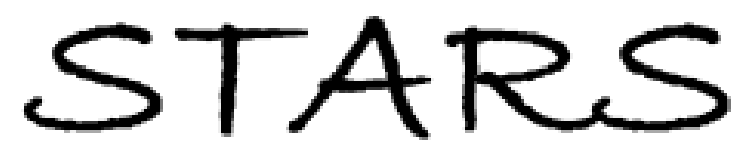

University of Central Florida

STARS

$1-1-2003$

\title{
Thickness dependence of the magnetic hysteresis of NiFe-31\% films as a function of an applied isotropic in-plane stress
}

Claude Garrett

Patrick Holland

Wilhelmus J. Geerts

Dustin Ragan

Archana Dubey

University of Central Florida

See next page for additional authors

Find similar works at: https://stars.library.ucf.edu/facultybib2000

University of Central Florida Libraries http://library.ucf.edu

This Article is brought to you for free and open access by the Faculty Bibliography at STARS. It has been accepted for inclusion in Faculty Bibliography 2000 s by an authorized administrator of STARS. For more information, please contactSTARS@ucf.edu.

\section{Recommended Citation}

Garrett, Claude; Holland, Patrick; Geerts, Wilhelmus J.; Ragan, Dustin; Dubey, Archana; Rios, Steve; and Bandyopadhyay, Anup K., "Thickness dependence of the magnetic hysteresis of NiFe- $31 \%$ films as a function of an applied isotropic in-plane stress" (2003). Faculty Bibliography 2000s. 3772.

https://stars.library.ucf.edu/facultybib2000/3772

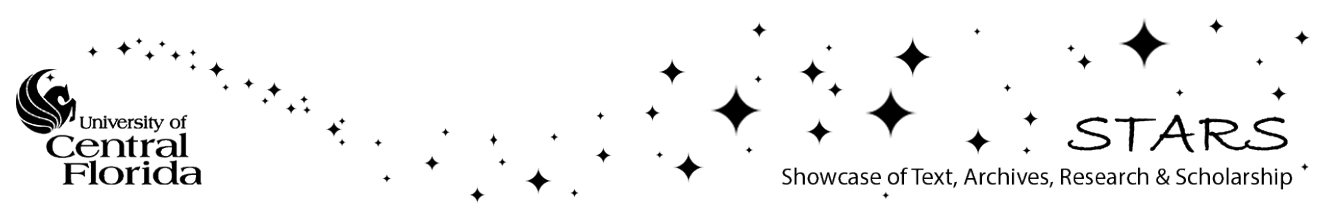




\section{Authors}

Claude Garrett, Patrick Holland, Wilhelmus J. Geerts, Dustin Ragan, Archana Dubey, Steve Rios, and Anup K. Bandyopadhyay 


\section{Thickness dependence of the magnetic hysteresis of NiFe-31\% films as a function of an applied isotropic in-plane stress}

Cite as: Journal of Applied Physics 93, 8624 (2003); https://doi.org/10.1063/1.1558242

Published Online: 09 May 2003

Claude Garrett, Patrick Holland, Wilhelmus J. Geerts, Dustin Ragan, Archana Dubey, Steve Rios, and Anup K. Bandyopadhyay

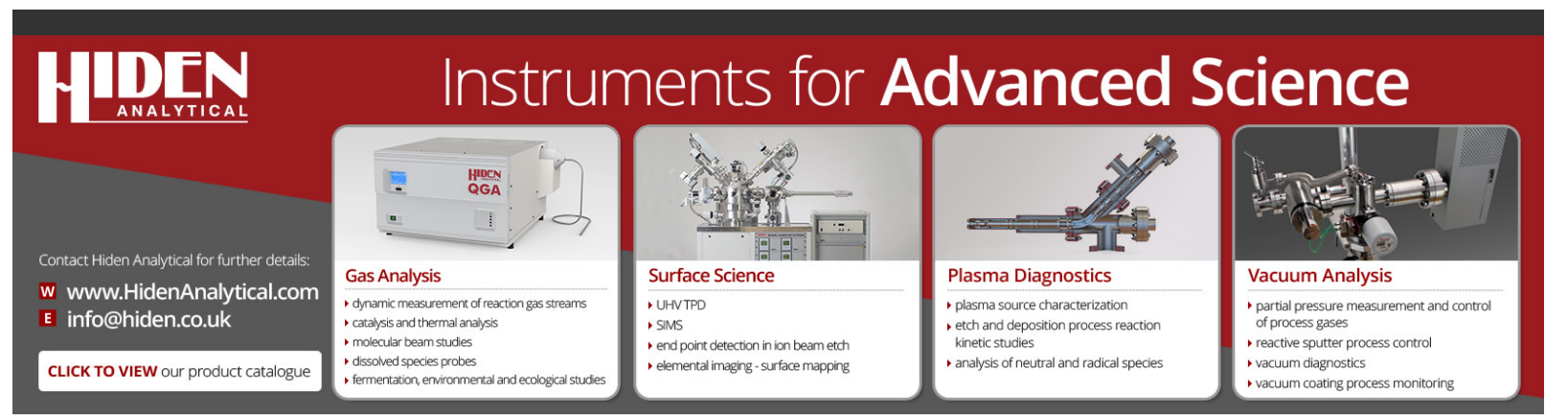




\title{
Thickness dependence of the magnetic hysteresis of NiFe-31\% films as a function of an applied isotropic in-plane stress
}

\author{
Claude Garrett, Patrick Holland, and Wilhelmus J. Geerts ${ }^{a)}$ \\ Department of Physics, Southwest Texas State University, 601 University Drive, San Marcos, Texas 78666 \\ Dustin Ragan \\ Department of Physics, Trinity University, San Antonio, Texas 78212 \\ Archana Dubey \\ Department of Physics, University of Central Florida, Orlando, Florida 32816
}

Steve Rios and Anup K. Bandyopadhyay

Department of Physics, Southwest Texas State University, 601 University Drive, San Marcos, Texas 78666

(Presented on 15 November 2002)

\begin{abstract}
The magnetic hysteresis of dc magnetron-sputtered $\mathrm{Ni}_{69} \mathrm{Fe}_{31}$ films that were sandwiched between titanium layers was investigated as a function of an externally applied isotropic in-plane strain. The hysteresis curves were measured with a Kerr magnetometer that monitored the longitudinal Kerr ellipticity as a function of the in-plane magnetic field. The strain was created by bending the samples in two dimensions using a pressure cell. Measurements were performed on films with different thicknesses. The magnetoelastic properties appeared to be much smaller for films with a thickness of $100 \mathrm{~nm}$ than for films with a thickness of 288 or $500 \mathrm{~nm}$. This might be due to a change of the domain wall pinning, or a change of the domain wall density as a function of the film thickness. Measurements under compressive isotropic in-plane stress were shown to be possible by flipping the sample in the pressure holder and measuring through the glass substrate. The Faraday effect and stress-induced birefringence in the glass substrate did not hinder the accumulation of noiseless hysteresis data. The magnetoelastic effects appeared to be different for positive and negative values of the applied stress. (C) 2003 American Institute of Physics. [DOI: 10.1063/1.1558242]
\end{abstract}

\section{INTRODUCTION}

When applying magnetic materials in sensors or actuators a good understanding of the magnetoelastic properties of thin ferromagnetic films is important; manufacturing induced stress caused by dicing, microstructuring, or packaging can strongly influence yields. Furthermore, thermally induced stress-relief over the lifetime of a device may cause reliability problems. ${ }^{1}$ A better understanding of the magnetoelastic properties is also relevant for the application of thin films in pressure, force, acceleration, and stress sensors where effects are maximized.

The magnetoelastic properties of thin films differ from those of bulk materials. Callegaro et al., ${ }^{2}$ for example, showed that the influence of stress on the coercivity of electrodeposited nickel films was opposite to that of bulk material. In magnetoelastic investigations, one normally applies a stress by bending the thin film in one direction. This causes a uniaxial anisotropy axis in the plane of the film that can considerably change the magnetization pattern. In our experiments we tried to keep a high degree of symmetry by using circular substrates and by bending the sample in two directions. No in-plane anisotropy axis that will directly influence the domain pattern was induced.

\footnotetext{
a) Author to whom correspondence should be addressed; electronic mail: wjgeerts@swt.edu
}

With this technique we recently ${ }^{3}$ investigated the magnetoelastic properties of $500-\mathrm{nm}$-thick $\mathrm{NiFe}-31 \%$ as a function of an isotropic tensile strain $(e)$ and found that the coercivity largely depends on $e$ with a $\Delta H_{c} / \Delta e$ as high as $1.5 \times 10^{4} \mathrm{Oe}$. In this paper we present the results of a study on how these magnetoelastic effects differ as a function of the film thickness. We also report, for the first time, on data measured while applying a compressive isotropic in-plane stress.

\section{EXPERIMENTAL PROCEDURE}

The films were prepared by dc magnetron sputtering on carefully cleaned circular shaped glass microscope slides. No attempt was made to adjust the deposition parameters to create stress free films. More details on the substrate cleaning and deposition procedures can be found in Ref. 3. The chemical composition of the samples was measured by energy dispersive spectroscopy and was determined to be $\mathrm{Ni}_{69} \mathrm{Fe}_{31}$. We used a Bede D1 x-ray diffractometer to determine the crystal structure of our films. The $x$-ray data showed a large fcc (111) peak in the $\theta-2 \theta$ plot from which we conclude that our films have a fcc crystal structure with a $\langle 111\rangle$ texture (see Fig. 1). The $2 \mathrm{~nm}$ Ti seed layer increases the adhesion of the NiFe film to the glass substrate and the 2 $\mathrm{nm}$ Ti cap layer prevents the film from oxidizing. Both the seed and cap layer are much thinner than the penetration depth of the HeNe light $(\lambda / 4 \pi k=17 \mathrm{~nm}$ at $2 \mathrm{eV}) .{ }^{4}$ Another 


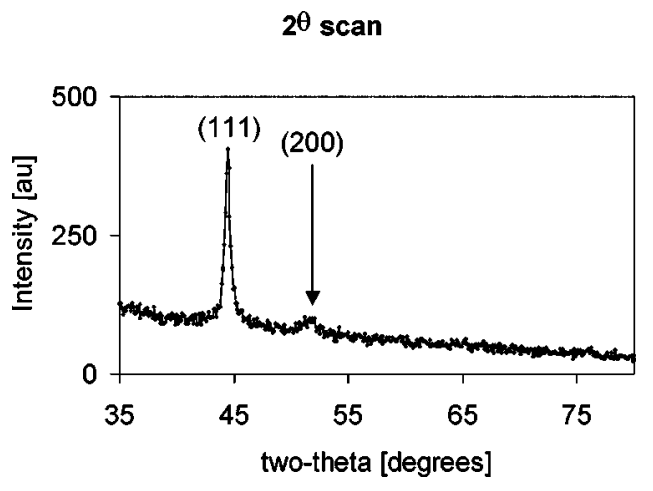

FIG. 1. Two-theta scan of $\mathrm{Ni}_{69} \mathrm{Fe}_{31}$.

advantage of using a Ti seed layer is the improvement of the chemical homogeneity throughout the thin film as shown by Takahashi et al. ${ }^{5}$ Additional samples were prepared without a seed layer to investigate how the Ti underlayer influences the magnetic properties.

The in-plane magnetic hysteresis curves were measured with a Kerr magnetometer as a function of an externally applied isotropic in-plane stress. This stress was applied by bending the samples in two dimensions using a pressure cell similar to the one developed by Callegaro et al. ${ }^{6}$ More details on the experimental setup can be found in Ref. 3. Measurements were performed with the thin film side up (tensile stress) and with the glass substrate side up (compressive stress). By monitoring the Kerr ellipticity as a function of the field, contributions of the Faraday effect in the glass substrate could be avoided. It also appeared that possible birefringence caused by the stresses in the microscope slide did not hinder the measurements. All measurements were performed in the longitudinal mode, i.e., the magnetic field parallel to the plane of incidence. The diameter of the beam was approximately $1 \mathrm{~mm}$.

An additional experiment was performed on a NiFe sample directly deposited on a 1 in. silicon wafer (wafer thickness $=0.3 \mathrm{~mm}$ ). By agitating this sample ultrasonically in acetone, the thin film could be removed from the substrate. The in-plane hysteresis curve of the as-deposited film and the removed free-standing film were measured by Kerr magnetometer and vibrating sample magnetometer (VSM).

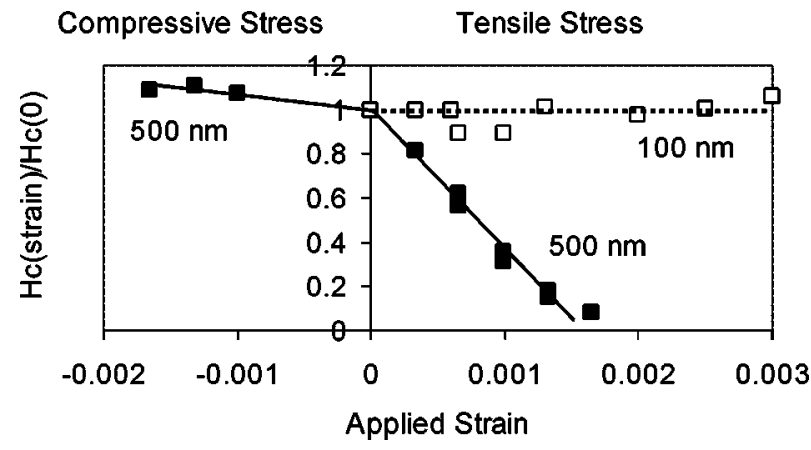

FIG. 2. Relative change of the coercivity as a function of an externally applied isotropic in-plane strain.
TABLE I. Samples: \#= sample name, $t=$ film thickness.

\begin{tabular}{lccc}
\hline \hline$\#$ & $t(\mathrm{~nm})$ & Structure & Coercivity $(\mathrm{Oe})$ \\
\hline 1 & 500 & $\mathrm{Glass} / \mathrm{Ti}(2 \mathrm{~nm}) /$ & $H_{c}($ Kerr $)=18$ \\
& & $\mathrm{NiFe} / \mathrm{Ti}(2 \mathrm{~nm})$ & $H_{c}(\mathrm{VSM})=16.2$ \\
2 & 100 & $\begin{array}{c}\mathrm{Glass} / \mathrm{Ti}(2 \mathrm{~nm}) / \\
\mathrm{NiFe} / \mathrm{Ti}(2 \mathrm{~nm})\end{array}$ & $H_{c}($ Kerr $)=4.5$ \\
& & $\mathrm{Glass} / \mathrm{NiFe}$ & $H_{c}(\mathrm{VSM})=0.6$ \\
4 & 528 & $\mathrm{Si}(300 \mu \mathrm{m}) /$ & $H_{c}($ KerrAsSput $)=1.5$ \\
& 288 & $\mathrm{NiFe}$ & $H_{c}($ KerrFreeStand $)=18.5$ \\
\hline \hline
\end{tabular}

\section{RESULTS AND DISCUSSION}

The coercivity and structures of the films are given in Table I. The VSM coercivity is close to the Kerr coercivity. This suggests that our films are very homogeneous. The films deposited directly on the glass showed a lower coercivity than the one sputtered on a Ti seed layer. It is very well known that a Ti seed layer can increase the size of the crystal grains and promote a strong $\langle 111\rangle$ texture of various types of materials. ${ }^{7}$ Since the coercivity in the NiFe also strongly depends on the crystal defect density, it is not possible to unambiguously explain the mechanism of the observed coercivity increase without further investigations. The observed increases are, however, significant and are also observed by others on different materials. ${ }^{8}$

Figure 2 shows the relative change in the coercivity $\left[H_{c}(e) / H_{c}(0)\right]$ as a function of the applied strain for samples 1 and 2 . The strain-coercivity relation is nonsymmetrically around the origin. Furthermore it depends on the film thickness. Callegaro et al. observed similar effects on uniaxial stressed nickel samples.

Figure 3 shows the in-plane hysteresis curves of sample 4 before and after it is removed from the silicon substrate. The free-standing film shows a much larger coercivity. The data suggest that the as-sputtered film is highly stressed and when removed from the substrate relaxes. We measured the curvature of a sample with similar thickness by stylus profilometer and could not find any bending of the substrate within the measurement error. 800-nm-thick films however

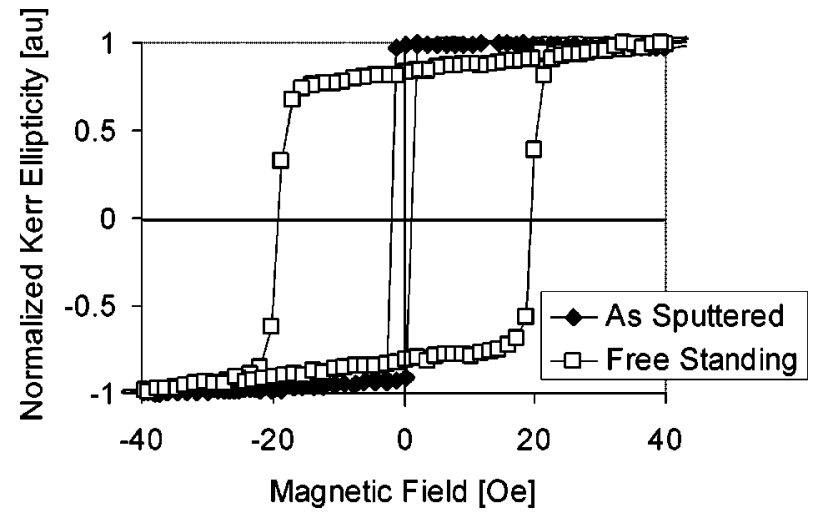

FIG. 3. In-plane Kerr hysteresis of an as-sputtered and a free-standing 288 nm thick NiFe film. 
were able to bend the silicon substrate, while 1300-nm-thick films would spontaneously peal off the silicon wafer within two weeks of deposition.

\section{CONCLUSIONS}

Our $\mathrm{Ni}_{69} \mathrm{Fe}_{31}$ films have an in-plane anisotropy. The positive magnetostriction is not large enough to push the magnetic moment out of the film plane for the negative applied stresses as was observed for nickel, ${ }^{3,6}$ but does decrease the $d H_{c}(e) / d e$ as shown in Fig. 2. Assuming that the main reversal mechanism in our films is domain wall motion the coercivity $\left(H_{c}\right)$ depends on the pinning of the domain walls. A pinning site is characterized by a local minimum of the domain wall energy. If $\gamma$ is the domain wall energy, $x$ is the position of the domain wall, $M_{s}$ is the saturation magnetization, and $\mu_{0}$ is the permeability in vacuum, we can write for $H_{c}: 9,10$

$$
H_{c} \approx \frac{1}{2 \mu_{0} M_{s}}\left(\frac{\partial \gamma}{\partial x}\right)_{\max } .
$$

Our data suggest that $(\partial \gamma / \partial x)_{\text {max }}$ depends on the applied stress to the sample and on the film thickness. The former is clear if we realize that the domain wall energy depends on the applied stress to the sample $(\sigma)$, i.e.,

$$
\gamma=4 \sqrt{A\left(K_{1}+\lambda_{s} \sigma\right)},
$$

where $A$ is the exchange constant, $K_{1}$ is the crystal anisotropy, and $\lambda_{s}$ is the magnetostriction coefficient. The thickness dependence of $(\partial \gamma / \partial x)_{\max }$ might originate from a surface or interface crystal anisotropy term, ${ }^{11}$ a thickness dependence of the magnetostriction, ${ }^{12,13}$ a thickness dependence of the defect density, a thickness dependence of domain wall width and density, or a change of the type of domain walls for the thicker films. In thicker films Bloch walls replace Neel walls. The latter are characterized by a rotation of the magnetization in the plane of the film. Since an applied stress will not change a lot in the plane of the film, smaller magnetoelastic effects are plausible for films with Neel walls. Domain studies need to be performed to reveal the exact nature of the observed effects. Experiments to study the magnetostriction as a function of the film thickness and the magnetoelastic properties of patterned thin films are in preparation.

\section{ACKNOWLEDGMENTS}

The authors would like to acknowledge support from the Division of Materials Research of NSF under Grant No. 0075372. This work is furthermore supported by an award from Research Corporation. The authors would like to thank Jett Hendrix of SWT for his comments on this manuscript and Dr. Carlos Gutierrez of SWT for his help with interpreting the EDS and x-ray data.

${ }^{1}$ W. van Drent (private communication).

${ }^{2}$ L. Callegaro and E. Puppin, Appl. Phys. Lett. 68, 1279 (1996).

${ }^{3}$ P. Holland, M. Kempton, D. Ragan, S. Rios, A. Bandyopadhyay, A. Dubey, and W. Geerts, J. Magn. Magn. Mater. 250, L1 (2002).

${ }^{4}$ D. W. Lynch, C. G. Olson, and J. H. Weaver, Phys. Rev. B 11, 3617 (1975).

${ }^{5}$ S. Takahashi, K. Yamakaw, N. Honda, K. Ouchi, and S.-i. Iwasaki, J. Magn. Soc. Jpn. 18, 385 (1994).

${ }^{6}$ L. Callegaro, D. Petrali, and E. Puppin, Rev. Sci. Instrum. 68, 1796 (1997).

${ }^{7}$ P. Bayle-Guillemaud, A. K. Petford-Long, T. X. Anthony, and J. A. Brug, IEEE Trans. Magn. 32, 4627 (1996).

${ }^{8}$ P. Perera, A. G. Ayala, and C. J. Gutierrez, J. Appl. Phys. 85, 6121 (1999).

${ }^{9}$ Ferromagnetic Materials: Structure and Properties, edited by R. A. McCurrie (Academic, London, 1994), ISBN: 0-12-482495-1.

${ }^{10}$ I. J. Garshelis, J. Appl. Phys. 73, 5629 (1993).

${ }^{11}$ L. Neel, J. Phys. Radium 15, 225 (1954).

${ }^{12}$ O. Song, C. A. Ballentine, and R. C. O’Handley, Appl. Phys. Lett. 64, 2593 (1994)

${ }^{13}$ M. P. Hollingworth and M. R. Gibbs, J. Appl. Phys. 93, 8737 (2003), these proceedings. 